\title{
The Effect of Thermal Expansion in a Dry Clutch on Launch Control ${ }^{\star}$
}

\author{
Andreas Myklebust* Lars Eriksson* \\ * Vehicular Systems, Electrical Engineering Department, Linköping \\ University, 58183 Linköping Sweden, (e-mail: \\ firstname.lastname@liu.se)
}

\begin{abstract}
A dry clutch model with thermal dynamics is added to a driveline model of a heavyduty truck equipped with an automated manual transmission. The model captures driveline oscillations and can be used to simulate how different clutch-control strategies affect vehicle performance, drivability and comfort. Parameters are estimated to fit a heavy-duty truck and the complete model is validated with respect to shuffle, speed trajectory, clutch torque and clutch lock-up/break-apart behavior. The model shows good agreement with data. Furthermore the model is used to study the effect of thermal expansion in the clutch on launch control. It is shown that the effect of thermal expansion, even for moderate temperatures, is significant in launch control applications.
\end{abstract}

\section{INTRODUCTION}

Increasing demands on comfort, performance, and fuel efficiency in vehicles lead to more complex transmission solutions. Historically high efficiency was best met with a classical Manual Transmission and comfort with a classical Automatic Transmission. The Automated Manual Transmission (AMT) is one way to combine the best from two worlds. An important part in an AMT is clutch control that has a profound effect on vehicle performance. Therefore it is of importance to know the torque transmitted in the clutch with high precision. Models have come to play an important role in estimation and control of the transmitted torque, since torque sensors are expensive.

A sketch of a dry single-plate clutch is found in Fig. 1, while in-depth explanations are found in for example Mashadi and Crolla [2012] and Vasca et al. [2011]. In clutch-modeling literature a wide range of models are proposed. The most simple models have a clutch torque that is assumed to be a controllable input, see for example Dolcini et al. [2008], Garofalo et al. [2002]. These models rely on the assumption that there is perfect knowledge of how the clutch behaves. More advanced models include submodels for slipping and sticking torques. For example a LuGre model is used in Dolcini et al. [2005] and a Karnopp model in Bataus et al. [2011]. The former is a one-state model that captures stick-slip behavior, varying breakaway force, Stribeck effect, and viscous friction. The latter simply applies a dead-zone around zero speed to ease the simulation of stick-slip behavior.

Models for the transmittable clutch torque during slipping commonly use a function with the following structure,

$$
M_{\text {trans,k }}=\operatorname{sgn}(\Delta \omega) \mu R_{e} F_{N}
$$

where $\Delta \omega$ is the clutch slip (speed), $\mu$ the friction coefficient, $R_{e}$ the effective radius and $F_{N}$ the clamping

\footnotetext{
* Vinnova industrial excellence center LINK-SIC and Scania CV have supported this work.
}

(normal) force. In these models $F_{N}$ is often either given as input or a static nonlinear function of clutch position, $x$, i.e. $F_{N}=F_{N}(x)$, see for example Vasca et al. [2011], Glielmo and Vasca [2000]. Furthermore a graph with speed dependency of the normal force is shown in Hong et al. [2012]. In Dolcini et al. [2010] that speed dependency is said to be due to centrifugal forces acting on the springs in the clutch. Mashadi and Crolla [2012], Moon et al. [2004] reports of hysteresis in the diaphragm spring, that could lead to hysteresis in the normal force. In Mattiazzo et al. [2002] a temperature and wear dependency of the normal force/bearing position characteristics is shown.

Concerning the other model components it is generally recognized that $\mu$ can depend on temperature, slip speed, and wear and that $R_{e}$ can depend on temperature and wear as well, see Velardocchia et al. [1999]. In Vasca et al. [2011] a slip speed dependency of $\mu R_{e}$ is shown, this was especially pronounced for slip speeds below $\sim 100$ RPM.

It can be difficult to separate which parameter in (1) that is the reason for a change in $M_{\text {trans,k }}$. Therefore the clutch torque is often studied as a lumped model. In Velardocchia et al. [1999] $M_{\text {trans,k }}$ is seen to decrease with temperature and $\Delta \omega$. However there are large variations with wear. In Ercole et al. [2000] $M_{\text {trans,k }}$ initially decreases with temperature for low temperatures and then increases for medium and high temperatures. Similarly there are variations with wear and in addition temperature-torque hysteresis are reported. In Velardocchia et al. [2000], Wikdahl and Ågren [1990] and Myklebust and Eriksson [2012b] temperature models are established, but only Myklebust and Eriksson [2012b] includes the effect of the temperature on $M_{\text {trans }}$.

When modeling the rest of the driveline for (clutch) control purposes it is common to include one or more flexibilities, Pettersson [1997], Garofalo et al. [2002], Fredriksson and Egardt [2003], Moon et al. [2004], Crowther et al. [2004], Lucente et al. [2007], Dolcini et al. [2008] 


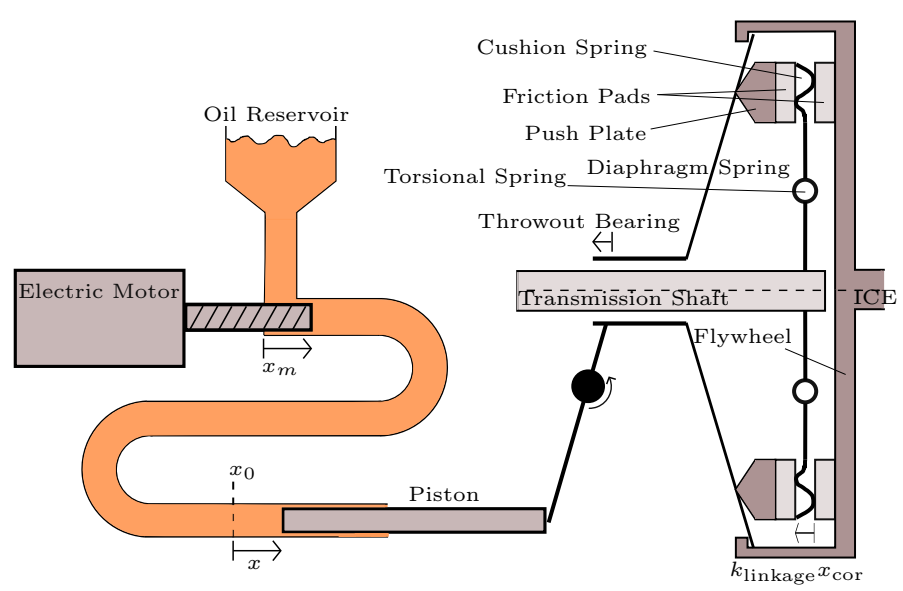

Fig. 1. A sketch over the actuator and dry single-plate pull-type clutch installed in the experiment vehicle.

Here yet another driveline model with focus on the clutch and the control of it is presented and validated. The contribution lies in that here the thermal dynamics of the clutch are included in the model. Particularly the significance to launch performance of including the thermal part is shown.

\section{DRIVELINE MODEL}

In order to evaluate the quality of a certain launch control, a longitudinal model of the heavy-duty truck in question is required. The model has to capture important dynamics in the driveline and how they make the truck shuffle. One such model is found in Myklebust and Eriksson [2012a]. It is used here with one modification, the clutch model is replaced with the more advanced model from Myklebust and Eriksson [2012b].

An overview of the model is seen in Figure 2. There the different parts of the model, Internal Combustion Engine (ICE), clutch, gearbox, propeller shaft, final drive, drive shafts and vehicle dynamics, can be seen as well as where the flexibilities are located. Next, a quick review of the model equations are given.

$$
\frac{1}{2} F_{d r} \longrightarrow
$$

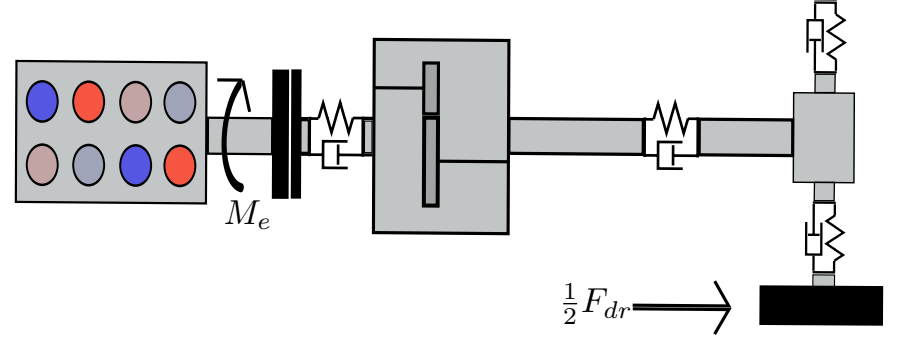

Fig. 2. Sketch of the driveline model. Modeled parts and locations of flexibilities are visible.

If not otherwise stated the nomenclature follows this system: $\theta=$ angle, $\omega=\dot{\theta}, v=$ velocity, $r=$ radius, $T=$ temperature $M=$ torque, $F=$ force, $P=$ power, $c=$ damping or vehicle dynamics coefficient, $k=$ spring coefficient, $b=$ viscous friction coefficient, $J=$ inertia, $i=$ gear ratio, and $x=$ clutch piston position. These quantities are often equipped with subscripts, $e=$ engine, $f w=$ flywheel, $c=$ clutch transmission side, $t=$ transmission, $p=$ propeller shaft, $f=$ final

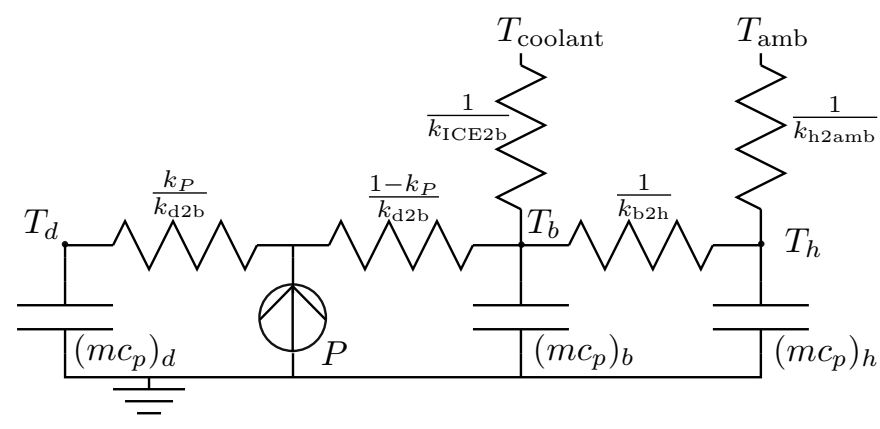

Fig. 3. An electrical analogy of the temperature model (3)(5).

drive, $d=$ drive shaft, $w=$ wheel, $i=$ gear number, and $\mathrm{amb}=$ ambient.

\subsection{Internal Combustion Engine}

The ICE produces the engine torque, $M_{e}$, that is given as model input. Note that this is the net (brake) torque of the ICE, e.g. $M_{e}=0$ with open clutch will keep the engine speed constant.

\subsection{Clutch}

The explanation of the clutch model is split into three parts, the friction and temperature dynamics, the mode changes between locked and slipping, and the torsional springs. An overview of the studied clutch is seen in Fig. 1.

Clutch Friction The natural output from the actuator is the clamping force, $F_{N}$. However $F_{N}$ is not measurable, therefore it is directly recalculated into a transmittable torque, $M_{\text {trans }}=k \mu F_{N}$, that is used as actuator output. The shape of the torque transmissibility curve is described by a third order polynomial, Dolcini et al. [2010], Myklebust and Eriksson [2012b]

$$
M_{\mathrm{ref}}\left(x_{\mathrm{ref}}\right)= \begin{cases}a\left(x_{\mathrm{ref}}-x_{\mathrm{ref}, \text { ISP }}\right)^{3}+ & \\ +b\left(x_{\mathrm{ref}}-x_{\mathrm{ref}, \text { ISP }}\right)^{2}, & \text { if } x_{\mathrm{ref}}<x_{\mathrm{ref}, \text { ISP }} \\ 0, & \text { if } x_{\mathrm{ref}} \geq x_{\mathrm{ref}, \text { ISP }}\end{cases}
$$

where $x_{\text {ISP }}$ (Incipient Sliding Point) is the kiss point. The exact value of $x_{\text {ISP }}$ can be difficult to find since the transmissibility curve is very flat around $x_{\text {ISP }}$. However it is only important to find a $x_{\text {ISP }}$ that gives a good curve fit, for a certain temperature, $T_{\text {ref }}$, as errors will be small near $x_{\text {ISP }}$.

The clutch disc temperature, $T_{d}$, clutch body (flywheel and pressure plate) temperature, $T_{b}$, and the clutch housing temperature, $T_{h}$, have been modeled in order to explain the torque drift, see Fig. 5, due to temperature. An electrical analogy of the model is found in Fig. 3 and below are the equations.

$$
\begin{aligned}
\left(m c_{p}\right)_{b} \dot{T}_{b} & =k_{\mathrm{ICE} 2 \mathrm{~b}}\left(T_{\text {coolant }}-T_{b}\right)+k_{\mathrm{b} 2 \mathrm{~h}}\left(T_{h}-T_{b}\right)+ \\
& +k_{\mathrm{d} 2 \mathrm{~b}}\left(T_{d}-T_{b}\right)+k_{P} P \\
\left(m c_{p}\right)_{h} \dot{T}_{h} & =k_{\mathrm{b} 2 \mathrm{~h}}\left(T_{b}-T_{h}\right)+k_{\mathrm{h} 2 \mathrm{amb}}\left(T_{\mathrm{amb}}-T_{h}\right) \\
\left(m c_{p}\right)_{d} \dot{T}_{d} & =k_{\mathrm{d} 2 \mathrm{~b}}\left(T_{b}-T_{d}\right)+\left(1-k_{P}\right) P
\end{aligned}
$$

where,

$$
P=M_{\mathrm{trans}, k} \Delta \omega=M_{\mathrm{trans}, k}\left(\omega_{e}-\omega_{c}\right)
$$


The temperature model is connected to the transmitted torque through a change of the position $x_{\text {cor }}$, see Fig. 1, corresponding to the expansion of parts in the clutch. The expansion of the clutch body and disc as a function of temperatures is assumed linear.

$$
\begin{gathered}
\Delta x_{0}=\left(k_{\exp , 1}+k_{\exp , 2}\right)\left(T_{b}-T_{\text {ref }}\right)+k_{\exp , 2}\left(T_{d}-T_{b}\right) \\
x_{\text {cor }}=x-\Delta x_{0}
\end{gathered}
$$

The transmitted torque can now be calculated as,

$$
M_{\text {trans }, k}=M_{\text {ref }}\left(x_{\text {cor }}\right)
$$

Note that $x_{\text {cor }}$ increases with temperature which in turn makes $M_{\text {trans, } k}$ increase with temperature. The $k$ in the subscript stands for kinetic because the friction is modeled as coulomb friction with stick-slip behavior. Define $k_{\mu}$ as the ratio of the static friction coefficient over the kinetic. Then the maximum transmittable torque when sticking is:

$$
M_{\text {trans }, s}=k_{\mu} M_{\text {trans }, k}
$$

Lock-Up/Break-Apart Logic The clutch model has two modes, locked and slipping mode. While in locked mode, the clutch behaves as one rigid body, whereas during slipping the clutch consists of two bodies where each one has an angular velocity and position. The equations are:

Conditions for switching from slipping to locked mode:

$$
\begin{aligned}
\dot{\theta}_{e} & =\dot{\theta}_{c} \\
M_{\text {trans }} & \leq M_{\text {trans }, \mathrm{s}}
\end{aligned}
$$

Conditions for switching from locked to slipping mode:

$$
M_{\text {trans }} \geq M_{\text {trans,s }}
$$

Equations specific for the clutch in locked mode:

$$
\begin{aligned}
M_{e}-M_{c} & =\left(J_{e}+J_{\mathrm{fw}}+J_{c}\right) \ddot{\theta}_{e} \\
\dot{\theta}_{c} & =\dot{\theta}_{e} \\
M_{\mathrm{trans}} & =\frac{M_{e} J_{c}+M_{c}\left(J_{e}+J_{\mathrm{fw}}\right)}{J_{e}+J_{\mathrm{fw}}+J_{c}}
\end{aligned}
$$

Equations specific to the clutch in slipping mode:

$$
\begin{aligned}
M_{\mathrm{trans}} & =\operatorname{sgn}\left(\dot{\theta}_{e}-\dot{\theta}_{c}\right) M_{\mathrm{trans}, \mathrm{k}} \\
M_{e}-M_{\mathrm{trans}} & =\left(J_{e}+J_{\mathrm{fw}}\right) \ddot{\theta}_{e} \\
M_{\mathrm{trans}}-M_{c} & =J_{c} \ddot{\theta}_{c}
\end{aligned}
$$

Torsional Part The main flexibility of the clutch is in the torsion springs in the clutch disc. They are located on the vehicle side of the friction surfaces and can be modeled as a separate part.

The clutch torsional part is modeled as a torsional spring and damper.

$$
M_{c}=c_{c}\left(\dot{\theta}_{c}-\dot{\theta}_{t}\right)+k_{c}\left(\theta_{c}-\theta_{t}\right)
$$

\subsection{Transmission}

The transmission consists of some inertia, viscous friction and a gear ratio. Note that no synchronizers are modeled and the model can not engage neutral gear. Therefore gear shifting will be instantaneous. This is an acceptable approximation when the clutch is disengaged, since the transmission input side has low inertia compared to the rest of the vehicle.
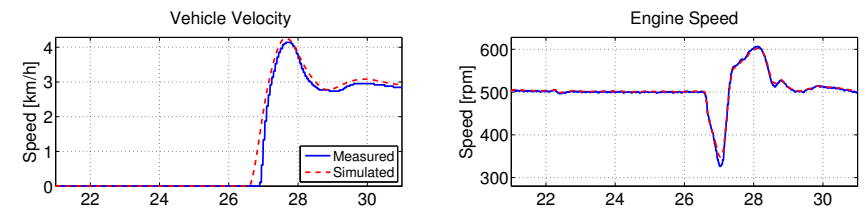

Gearbox Input Speed

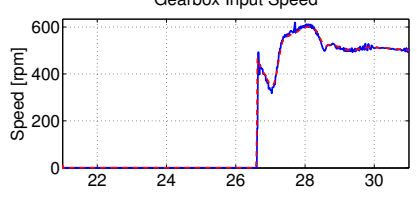

Gearbox Output Speed
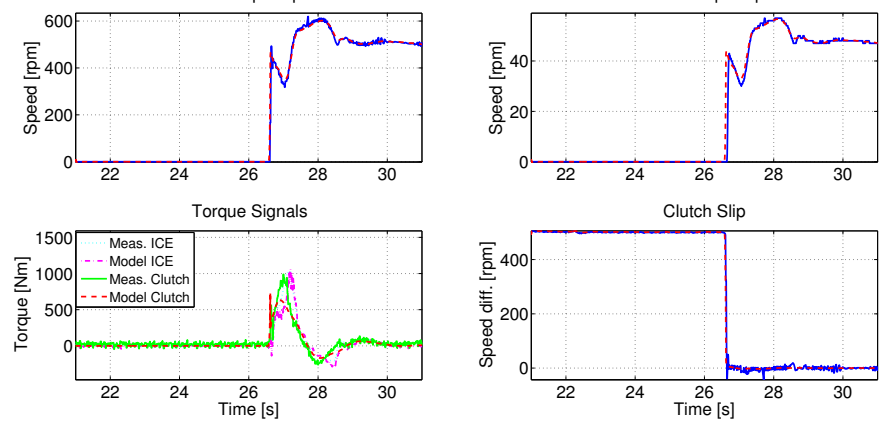

Fig. 4. A quick launch (clutch locks-up in less than $0.1 \mathrm{~s}$ ) with large oscillations was used to parameterize the driveline model. Both the real and modeled engines were under speed control. The measured output-shaft speed is lagging the model due to sensor dynamics.

With the states $\dot{\theta}_{p}, \theta_{p}$, and $\theta_{t}$ the equations become:

$$
\begin{aligned}
M_{t} & =M_{c} i_{t, i} \\
\left(J_{t, i}+J_{p}\right) \ddot{\theta}_{p} & =M_{t}-b_{t} \dot{\theta}_{p}-M_{p} \\
\dot{\theta}_{t} & =\dot{\theta}_{p} i_{t, i}
\end{aligned}
$$

\subsection{Propeller Shaft}

The flexible propeller shaft is modeled in the same way as the clutch flexibility, (20). The propeller-shaft equation is:

$$
M_{p}=c_{p}\left(\dot{\theta}_{p}-\dot{\theta}_{f}\right)+k_{p}\left(\theta_{p}-\theta_{f}\right)
$$

\subsection{Final Drive}

The final drive with differential is assumed to act symmetrically on the drive shafts. Therefore it can be modeled as the transmission but with fixed gear ratio, $i_{f}$, and inertia, $J_{f}$. With the states $\dot{\theta}_{d}, \theta_{d}$ the equations become:

$$
\begin{aligned}
\left(J_{p} i_{f}^{2}+J_{f}+J_{d}\right) \ddot{\theta}_{d} & =M_{p} i_{f}-b_{f} \dot{\theta}_{d}-M_{d} \\
\dot{\theta}_{f} & =\dot{\theta}_{d} i_{f}
\end{aligned}
$$

\subsection{Drive Shafts}

The driveline's main flexibility is in the drive shafts, which can, with a symmetrical differential, be modeled as the clutch flexibility, (20). The drive shaft equation is:

$$
M_{d}=c_{d}\left(\dot{\theta}_{f}-\dot{\theta}_{w}\right)+k_{d}\left(\theta_{f}-\theta_{w}\right)
$$

\subsection{Vehicle Dynamics}

The wheels and non-driveline parts that affect the longitudinal dynamics are modeled in this section. Tire dynamics are neglected and rolling condition is assumed. The wheels simply consists of a radius, $r_{w}$, an inertia, $J_{w}$ and a rolling resistance force, $F_{r} . F_{r}$ is multiplied with a smoothing function in order to improve performance of the simulation, Myklebust and Eriksson [2012a]. 
Model inputs that directly affect the vehicle dynamics are braking force and road-slope angle, $\alpha$ (in radians). The road-slope angle is used to calculate the gradient force that is added with the braking force, rolling resistance and aerodynamic drag.

With the states $v(\dot{v}=a)$ and $\theta_{w}$ the equations become:

$$
\begin{gathered}
F_{a}=\frac{1}{2} \rho_{a} c_{w} A_{f} v^{2}, \quad F_{g}=m g \sin (\alpha) \\
F_{r}=f(v)\left(c_{r 1}+c_{r 2}|v|\right) m g, \quad \dot{\theta}_{w}=v / r_{w} \\
\frac{M_{d}}{r_{w}}-\operatorname{sgn}(v)\left(F_{r}+F_{a}+F_{b}\right)-F_{g}= \\
=\frac{M_{d}}{r_{w}}-F_{d r}=\left(m+\frac{J_{w}+J_{d}}{r_{w}^{2}}\right) a
\end{gathered}
$$

Let torsions replace the states corresponding to angles, then the state vector is reduce to: $\omega_{e}, \omega_{c}, \theta_{c}-\theta_{t}, \omega_{p}$, $\theta_{p}-\theta_{f}, \omega_{d}, \theta_{d}-\theta_{w}, \omega_{w}, T_{b}, T_{h}$, and $T_{d}$. When the clutch is locked one state disappears, $\omega_{e}=\omega_{c}$

\section{PARAMETER ESTIMATION}

The driveline model and the clutch model have both been validated separately in their respective paper. However they have not been validated when put together and the lock-up/break-a-part detection has not been validated before. In order to do that a number of launches have been recorded. However these experiments have been carried out in a different truck than those used in the previous papers. Therefore the parameters have to be estimated. The driveline parameters have been estimated using a launch where the clutch has been closed rapidly (clutch is slipping less than $0.1 \mathrm{~s}$ ). This gives negligible clutch dynamics and large shuffle oscillations, which is appropriate when estimating the flexibilities and damping coefficients. The result can be seen in Fig. 4. Since it is difficult to do open-loop simulation of a system under feedback, the model also utilizes feedback. In the experiments the combustion engine is under speed control, therefore also the modeled engine is put under speed control. A PIcontroller with feedforward of the measured engine torque is used. However since the engine model is simply an inertia, the PI-controller performs better than the real controller. Therefore the measured engine speed has been used as reference, in order to capture the imperfections in the speed control and the inherent shuffle. The other driveline speeds follow the measurements well. The model is leading somewhat in the start due to sensor dynamics. There is some difference in the engine reported torque and the modeled engine torque, although it is hard to draw any conclusions from this, since the torque signal is inexact during transients. The parameterization seems good.

Next the clutch model needs to be parameterized. The experiment conducted to do this has consisted of ramping the clutch position back and forth while the truck has been kept stationary using the parking brake. The resulting data can be seen in Fig. 5. The torque drift due to temperature can clearly be seen. By applying (3)-(8) to the data, using the same parameters as in Myklebust and Eriksson [2012b], Fig. 6 is attained. There the ramps have converged to one curve and consequently these parameters work here too. The 3rd degree polynomial, (2), has been fitted to this curve using the least square method.

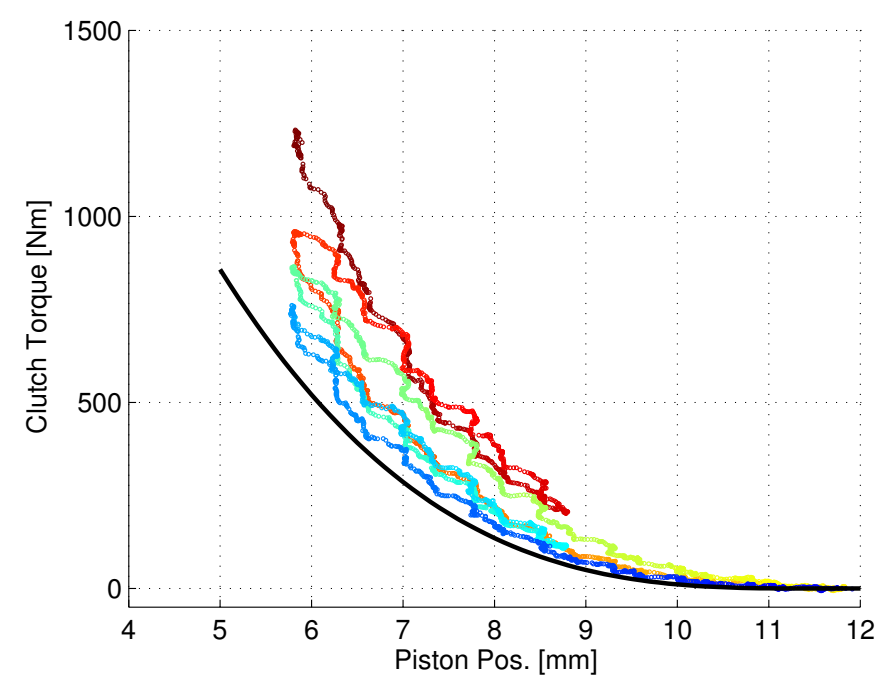

Fig. 5. The data used for parameterizing the clutch model. The torque drift with temperature can be clearly seen. The color indicates time (blue $=0 \mathrm{~s}, \mathrm{red}=45 \mathrm{~s}$ ).

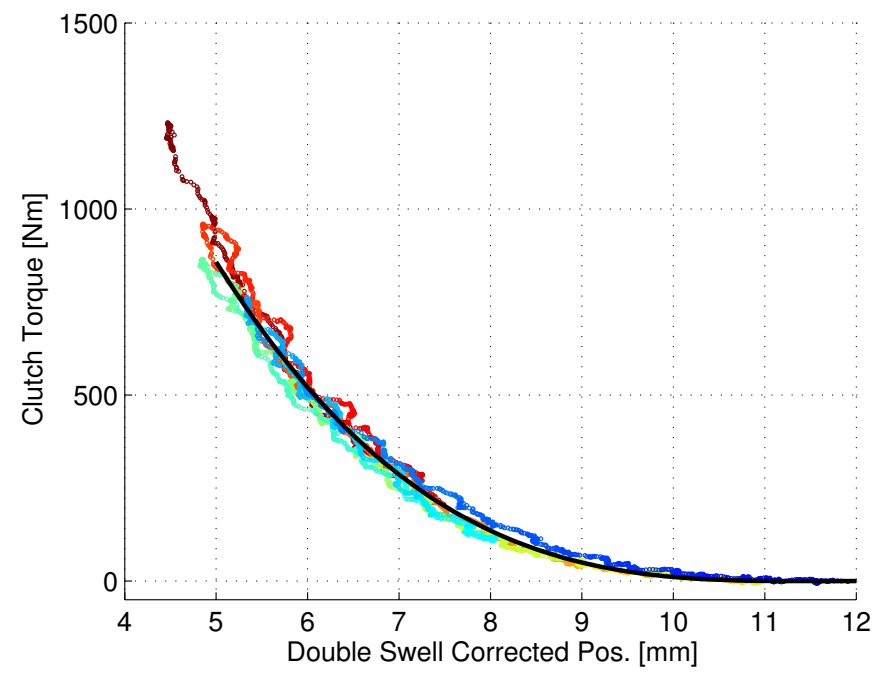

Fig. 6. Same as Fig. 5 but the position has been corrected for the thermal expansion using (8). All lines converge. The black line is the least square fit of the $3 \mathrm{rd}$ degree polynomial, (2).

\section{MODEL VALIDATION}

A number of launches have been performed in different gears in order to validate the complete model. Here two launches are shown, one in third gear, Fig. 7, and one in sixth gear, Fig. 8. In third gear there is some drift in vehicle speed with the consequence that the clutch locks up earlier in the model compared to the measurement. During the slipping phase the clutch torque has been modeled correctly, however after lock up the torque decreases due to the engine speed controller. When the engine speed reaches a set value the controller is switched off and torque is used as model input, since then the measurement is no longer under feedback. When the controller is switched off the drift naturally returns. Nevertheless the oscillations in the driveline are captured with respect to amplitude and frequency, although the attenuation in the model is a bit too high. In sixth gear the driveline speeds and 

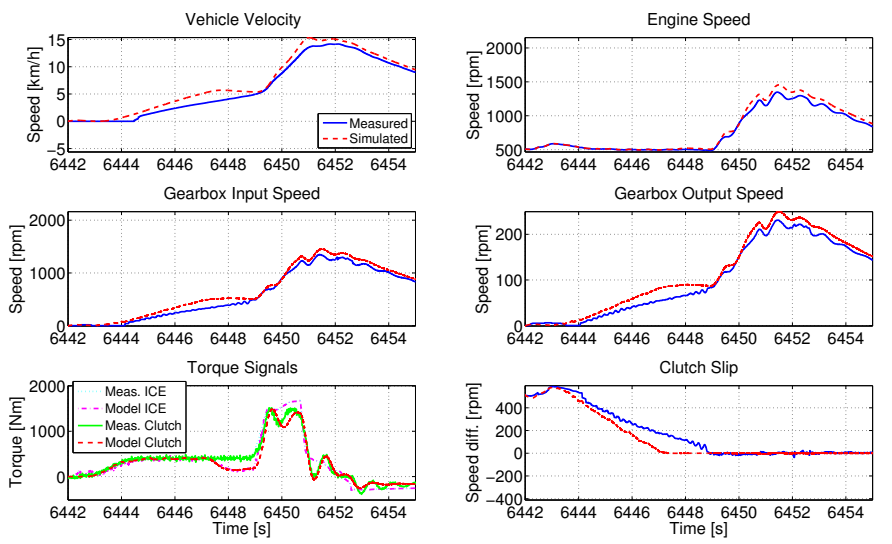

Fig. 7. Validation of the complete model in 3rd gear. Vehicle oscillations are captured and the clutch torque is correct (while slipping). The engine is under speed control when the speed is close to $500 \mathrm{RPM}$ and using torque as input otherwise.
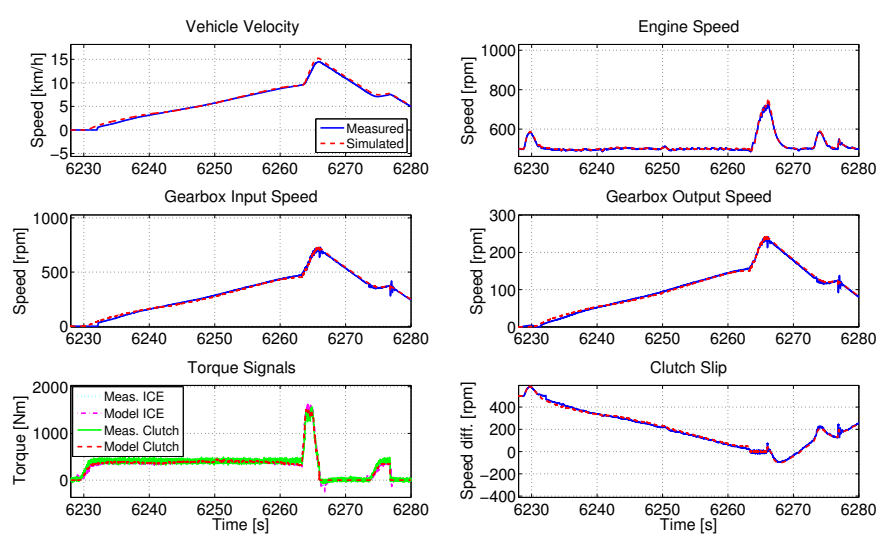

Fig. 8. Validation of the complete model in 6th gear. Vehicle motion and the clutch torque are modeled with high accuracy. The lock-up/break-apart logic can be seen to work as intended at $6263 / 6266$ s. When the torque transferred in the clutch changes between positive and negative some oscillations are seen in the measurement due to unmodeled backlash. The engine is under speed control.

clutch torque matches the measurement very well. As a result it is easy to see that the lock-up/break-apart logic makes mode switches at the correct time points. This is a further addition to the base-line model in Myklebust and Eriksson [2012a]. However when the clutch torque goes from positive to negative and vice versa there is some oscillations seen in the measurement due to backlash. These oscillation are naturally not captured in the model since the backlash is not modeled.

In conclusion both validations look fine and the model is suitable for investigating different clutch control strategies during launch and their effect on vehicle shuffle and performance.

\section{THERMAL EFFECT ON LAUNCHING}

This section highlights possible problems that can arise in clutch control during launch when not considering the thermal effects. Here two controllers taking requested
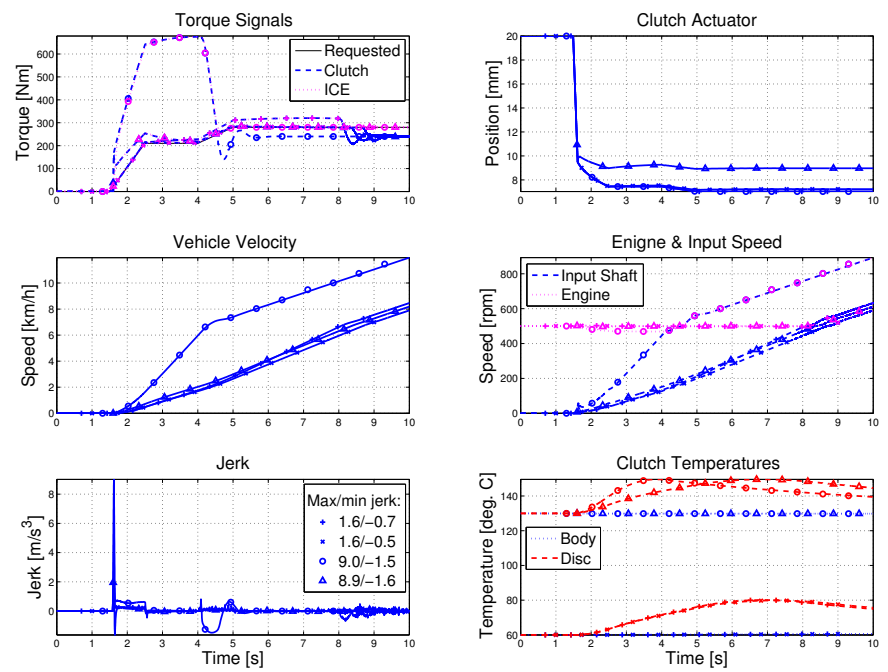

Fig. 9. A simulated launch in fourth gear for four different cases, cold clutch with open loop control marked with plus signs, cold clutch with closed loop control marked with crosses, warm clutch with open loop control marked with circles and warm clutch with closed loop control marked with triangles. Cold clutch means $\left[T_{b}, T_{h}, T_{d}\right]=[60,50,60]^{\circ} \mathrm{C}$ and warm means $\left[T_{b}, T_{h}, T_{d}\right]=[130,120,130]^{\circ} \mathrm{C}$. The cold closed loop case follows the reference torque closely whereas the cold open loop case has a slight drift due to heating of the clutch. The warm open loop case has a completely different speed trajectory that should correspond to a different accelerator input and, as in both warm cases, jerk levels are about five times higher.

torque as input are studied. This is a natural choice of input since it is common to use torque based driveline control, Heintz et al. [2001]. When so the driver intention over time can be interpreted as a reference torque trajectory. The first controller is the simplest possible, an open-loop controller consisting of an inversion of (2), the torque transmissibility curve at $60{ }^{\circ} \mathrm{C}$. The second controller uses the first controller as a feedforward part but in addition it has a PI-controller in order to utilize the engine torque for feedback. For both controllers the clutch is fully open when the torque request is zero and fully closed when there is no slip in the clutch. The clutch actuator is fast and exact and therefore it is modeled with a rate limiter of $82 \mathrm{~mm} / \mathrm{s}$ on $x$. The ICE directly gives the requested torque and has in addition a PI idle-speed controller in order to not stall the engine if the requested torque is too low. The simulations will be evaluated mainly from a comfort perspective and a measurement relating to comfort is jerk (time derivative of the vehicle acceleration). According to Zeng et al. [2013] the maximum jerk and minimum (negative) jerk are important comfort measures.

A requested torque trajectory has been chosen as follows; starts out at zero torque until $1.5 \mathrm{~s}$ where it is ramped up to $3 \%$ of maximum torque at $2.5 \mathrm{~s}$. It is kept there until $4 \mathrm{~s}$ when it is ramped further up to $4 \%$ at $5 \mathrm{~s}$ and kept there for the rest of the simulation. This trajectory is used as input to the simulation model together with no braking, no slope, fourth gear and 500 RPM as idle speed. Simulations are run for both controllers in cold, $\left[T_{b}, T_{h}, T_{d}\right]=[60,50,60]^{\circ} \mathrm{C}$, and warm, $\left[T_{b}, T_{h}, T_{d}\right]=$ 
$[120,110,120]^{\circ} \mathrm{C}$, conditions, four cases in total. The results are seen in Fig. 9. The maximum jerk levels can be seen to rise more than a factor of five when the clutch is warm. This is due to that the clutch controller overshoots the kiss point at $1.5 \mathrm{~s}$. The feedback controller also get a large negative jerk when it tries to compensate for the excessive torque due to incorrect kiss point. In the open loop case no such compensation is present and naturally the truck recieves a completly different speed trajectory although the driver input is the same. Furthermore the jerk is larger when the clutch locks-up in this case. The openloop controller for the cold case has a drift in torque due to heating of the clutch. Only the closed-loop controller for the cold case manages to follow the desired trajectory.

Even though the example in Fig. 9 utilizes simple clutchcontrol algorithms it highlights a problem that will be present in any controller that does not compensate for the thermal dynamics. In order to give a quick response to driver request the clutch needs to quickly move to the kiss point. If the kiss point is overshot large discomfort can arise, as seen in Fig. 9. An additional problem is that the large change in the torque transmissibility curve can put the controller in a situation it has not been tuned for, as demonstrated by the negative peak in the jerk for the closed loop controller in the warm case. Moreover it should be mentioned that the temperatures used in this example are normal, temperatures can even go above $200^{\circ} \mathrm{C}$.

A remedy for this problem is to estimate the temperature and compensate the clutch piston position for the expansion using the model in Myklebust and Eriksson [2012b].

\section{CONCLUSION}

A driveline model for vehicle shuffle and a clutch model including thermal effects have been merged together in order to simulate how different clutch-control strategies affect vehicle shuffle and performance. Parameters have been estimated to fit a heavy-duty truck and the complete model has been successfully validated, including the lockup/break-apart logic. The complete driveline model has been used to show the profound effect of thermal phenomenon in the clutch on launch control, even for moderate temperatures. The launch control example showcases the importance of incorporating a thermal model of the clutch in launch control applications.

\section{REFERENCES}

M. Bataus, A. Maciac, M. Oprean, and N. Vasiliu. Automotive clutch models for real time simulation. Proc. of the Romanian Academy, Series A, 12(2):109-116, 2011.

A Crowther, N Zhang, D K Liu, and J K Jeyakumaran. Analysis and simulation of clutch engagement judder and stick-slip in automotive powertrain systems. Proc IMechE,, Part D: J. of Automobile Engineering, 218 (12):1427-1446, December 2004.

P. Dolcini, C. Canudas de Wit, and H. Béchart. Improved optimal control of dry clutch engagement. Proc. of the 16th IFAC World Congress, 16(1), 2005.

P. Dolcini, C. Canudas de Wit, and H. Béchart. Lurch avoidance strategy and its implementation in AMT vehicles. Mechatronics, 18(1):289-300, May 2008.
P. J. Dolcini, C. Canudas de Wit, and H. Béchart. Dry Clutch Control for Automotive Applications. Advances in Industrial Control. Springer-Verlag London, 2010.

G. Ercole, G. Mattiazzo, S. Mauro, M. Velardocchia, F. Amisano, and G. Serra. Experimental methodologies to determine diaphragm spring clutch characteristics. In SAE Technical Paper: 2000-01-1151, March 2000.

J. Fredriksson and B. Egardt. Active engine control for gear shifting in automated manual transmissions. Int. J. of Vehicle Design, 32(3-4):216-230, 2003.

F. Garofalo, L. Glielmo, L. Iannelli, and F. Vasca. Optimal tracking for automotive dry clutch engagement. In 2002 IFAC, 15th Triennial World Congress, 2002.

L. Glielmo and F. Vasca. Optimal control of dry clutch engagement. In SAE Paper: 2000-01-083\%, March 2000.

N. Heintz, M. Mews, G. Stier, A. J. Beaumont, and A. D. Noble. An approach to torque-based engine management systems. In SAE Technical Paper: 200101-0269, March 2001.

S. Hong, S. Ahn, B. Kim, H. Lee, and H. Kim. Shift control of a 2-speed dual clutch transmission for electric vehicle. In 2012 IEEE Vehicle Power and Propulsion Conference, October 2012.

G. Lucente, M. Montanari, and C. Rossi. Modelling of an automated manual transmission system. Mechatronics, 17(1):73-91, November 2007.

B. Mashadi and D. Crolla. Vehicle Powertrain Systems. John Wiley \& Sons Ltd, first edition, 2012.

G. Mattiazzo, S. Mauro, M. Velardocchia, F. Amisano, G. Serra, and G. Ercole. Measurement of torque transmissibility in diaphragm spring clutch. In SAE Technical Paper: 2002-01-0934, March 2002.

S.E. Moon, M.S. Kim, H. Yeo, H.S Kim, and S.H Hwang. Design and implementation of clutch-by-wire system for automated manual transmissions. Int. J. Vehicle Design, 36(1):83-100, 2004.

A. Myklebust and L. Eriksson. Road slope analysis and filtering for driveline shuffle simulation. In 2012 IFAC Workshop on Engine and Powertrain Control, Simulation and Modeling, October 2012a.

A. Myklebust and L. Eriksson. Torque model with fast and slow temperature dynamics of a slipping dry clutch. In 2012 IEEE VPPC, October 2012b.

M. Pettersson. Driveline Modeling and Control. PhD thesis, Linköpings Universitet, May 1997.

F. Vasca, L. Iannelli, A. Senatore, and G. Reale. Torque transmissibility assessment for automotive dry-clutch engagement. IEEE/ASME transactions on Mechatronics, 16(3):564-573, June 2011.

M. Velardocchia, G. Ercole, G. Mattiazzo, S. Mauro, and F. Amisano. Diaphragm spring clutch dynamic characteristic test bench. In SAE Technical Paper: 199901-0737, March 1999.

M. Velardocchia, F. Amisano, and R. Flora. A linear thermal model for an automotive clutch. In $S A E$ Technical Paper: 2000-01-0834, March 2000.

A. Wikdahl and $\AA$. Ågren. Temperature distribution in a clutch. Master's thesis, Linköping University, 1990.

H. Zeng, Y. Lei, Y. Fu, Y. Li, and W. Ye. Analysis of a shift quality metric for a dual clutch transmission. In SAE Technical Paper: 2013-01-0825, April 2013. 